\title{
PESOS DE ATRIBUTOS COM BASE EM ORDENAÇÕES PARA COMPARAÇÃO DE PROCESSOS DE SOLDAGEM EM AÇO INOXIDÁVEL*
}

Alessandro de Castro Corrêa ${ }^{1}$ Cícero Alberto Pereira Belém Pereira ${ }^{2}$

Paulo D'Angelo Costa Assunção ${ }^{3}$

Danielle Cristina Gonzaga Corrêa ${ }^{4}$

Cláudia Canto de Souza Leão ${ }^{5}$

Arildomá Lobato Peixoto 6

\section{Resumo}

A comparação de processos de soldagem é uma tarefa complexa por envolver diversos atributos. A designação dos pesos dos atributos a serem avaliados é uma etapa fundamental na análise de problemas multicritérios; contudo, por vezes, a única informação que se obtém é a ordem de prioridade. O objetivo deste trabalho foi calcular os pesos das propriedades destinadas à comparação de processos de soldagem em aço inoxidável AISI 316L para o reparo de eixo naval com base na ordenação fornecida pelo tomador de decisão. Os atributos considerados foram taxa de deposição, energia de soldagem, rendimento econômico, diluição e relação reforço-largura. Os métodos de atribuição de pesos aplicados foram o de ponderação igual (Equal Weights), ordenação recíproca (Rank Reciprocal), somatória (Rank Sum), ordenação exponente (Rank Exponent) e o método do centroide (Rank Order Centroid). Para se verificar o efeito da escolha dos pesos estimados numa avaliação multicritério, avaliaram-se dados reais, aplicando-se a Ponderação Aditiva Simples (Simple Additive Weighting). Os resultados indicaram que diferentes atribuições de pesos podem alterar a posição das alternativas. Foi sugerido que a elaboração tabela ou gráficos com as distribuições de pesos pode ser útil para que o tomador decisão identifique a que melhor reflete as suas preferências.

Palavras-chave: Soldagem; Multicritério; Pesos; Aço Inoxidável.

\section{ORDER-BASED ATTRIBUTE WEIGHTING OF STAINLESS STEEL SHAFT REPAIR USING WELD OVERLAY}

\section{Abstract}

Comparing welding coating processes is a complex task due to various attributes involved. Weight assignment to attributes is a major step of multi-attribute decision analysis, however, there are times when the properties priority order is the only information available. The aim of this study was to calculate the properties weights in order to stainless steel AISI $316 \mathrm{~L}$ shaft repair using weld overlay based on the attributes rank provided by the decision maker. Five rank ordering criteria weighting methods were applied, the Equal Weights, the Rank Reciprocal Weights, Rank Sum Weights, Rank Exponent Weights and the Rank Order Centroid method. With the view of examining the estimated weight choice effect on multi-criteria evaluation, the Simple Additive Weighting method was applied to real data analysis. Results indicated that different weight assignments can change the alternative's positions. It has been suggested that the weights distribution could be provided in tables and charts, so these can be useful for the decision maker to identify the one which best reflects his preferences. 
Keywords: Welding; Multi-attribute; Weight; Stainless Steel.

1 Administrador, Doutor em Administração, Professor Titular, Programa de Pós-Graduação em Engenharia de Materiais (PPGEMAT/IFPA) e Programa de Pós-Graduação em Engenharia Industrial (PPGEI/UFPA), IFPA, Belém, Pará, Brasil.

2 Engenheiro Mecânico, Mestrando, Programa de Pós-Graduação em Engenharia Industrial (PPGEI), Instituto de Tecnologia, UFPA, Belém, Pará, Brasil.

3 Me. em Engenharia Mecânica, Doutorando na Universidade Federal do Para, UFPA, Belém, Pará, Brasil.

4 Administradora, Mestre em Administração, Professora, Curso de Administração, Faculdade IdealDeVry Brasil, Belém, Pará, Brasil.

5 Engenheira de Produção, Acadêmica de Mestrado, Programa de Pós-Graduação em Engenharia de Materiais (PPGEMAT), IFPA, Belém, Pará, Brasil.

6 Formação/graduação, titulação, cargo/função, setor/departamento, Instituição de trabalho e/ou estudo, cidade, estado e país.. 


\section{INTRODUÇÃO}

O processo decisório pode ser uma atividade complexa quando há diversas exigências a serem satisfeitas. No campo da engenharia, a maioria dos problemas são essencialmente multicritérios por envolverem vários objetivos [1].

Tal complexidade pode ser verificada quando se comparam alternativas de processos de soldagem como os de revestimentos destinados a reparos de eixos propulsores navais pela existência de várias propriedades que devem ser comparadas, podendo ocorrer que algum processo demonstre desempenho vantajoso em determinados aspectos, mas não em outros.

Nesse contexto, os Métodos Multicritérios de Tomada de Decisão (MCDM) podem ser ferramentas de assistência apropriadas uma vez que apoiam a escolha da melhor alternativa quando diversos atributos, por vezes conflitantes, são considerados simultaneamente [2]. Os problemas de engenharia aos quais vem sendo aplicados os MCDM são diversificados. Por exemplo, na seleção de materiais [3], no planejamento de experimento e otimização [4], na manutenção [5], em projetos [6], processos produtivos [7] e na análise de sistemas de custos [8]. Yoon e Hwang [2] explicam que os MCDM envolvem as seguintes etapas:

(a) determinação das alternativas a disposição do tomador de decisão e os atributos;

(b) determinação dos atributos, também denominados critérios ou objetivos, que são as características que descrevem as qualidades das alternativas;

(c) atribuição dos pesos aos atributos;

(d) cálculos de pontuação, esses realizados por um modelo escolhido conforme as peculiaridades do problema; $e$

(e) ordenação das alternativas.

Os pesos dos atributos devem refletir as preferências do decisor, normalmente a somatória dos diversos pesos totalizam um, o que corresponde a $100 \%$ da importância dos objetivos em análise. Há muitas maneiras de designar tais pesos, desde a informação de números pontuais subjetiva e diretamente fornecida, até em forma de intervalos simples ou com apoio de lógica fuzzy [2]; [9], [10] e [11]; todavia, pode ocorrer que, a princípio, o decisor não se sinta seguro de atribuir valores, mas saiba claramente a ordem de prioridade dos atributos para a aplicação em foco. Nesses casos, podem se empregar técnicas de cálculo dos pesos com base na informação contida na ordenação.

Os métodos de atribuição de pesos baseados em ordenações são os mais simples, uma vez que exigem somente que os tomadores de decisão organizem os atributos por ordem de importância.

Este trabalho tem o objetivo de calcular os pesos das propriedades destinados à comparação de processos de soldagem no reparo de eixo naval de aço inoxidável AISI 316L em situações em que o tomador de decisão é capaz de informar a ordem de prioridade dos atributos a serem avaliados.

\section{MATERIAIS E MÉTODOS}

As informações a respeito da prioridade das propriedades foram fornecidas por um engenheiro mecânico com mais de 10 anos de experiência profissional. Diante de uma lista pré-elaborada que the foi apresentada, foi solicitado que as ordenasse da 
mais importante a menos importante, podendo haver empates, considerando que se destinavam à avaliação de processos de soldagem para reparo de eixo de propulsor naval de aço inoxidável AISI 316L.

Os processos de soldagem em aço inoxidável para o material do eixo naval cujas propriedades serão avaliados estão listados no Quadro $1 \mathrm{com}$ a respectiva codificação

Quadro 1. Processos de soldagem a serem avaliados

\begin{tabular}{|l|l|l|}
\hline I & Processo & Código \\
\hline 1 & SMAW (AWS E 309 L) & SMAW \\
\hline 2 & GMAW automatizado AWS AR 307 & GMAW 1 \\
\hline 3 & GMAW manual AWS AR 307 & GMAW 2 \\
\hline 4 & GMAW manual AWS AR 309 L & GMAW 3 \\
\hline 5 & GMAW automatizado AWS AR 309 L & GMAW 4 \\
\hline 6 & GMAW automatizado AWS AR 309 L com adição de nióbio & GMAW 5 \\
\hline
\end{tabular}

Para o levantamento de indicativos, foram utilizados dois processos de soldagem (SMAW e GMAW) com ramificações, ou seja, automatizado, manual e automatizado com adição de Nióbio ( $\mathrm{Nb}$ ), com isso seis corpos de prova foram confeccionados, onde, conforme parâmetros estabelecidos, foi calculado e exposto a Taxa de deposição (TD), Energia de soldagem (E), Rendimento econômico (E), Diluição (D) e Relação reforço-largura (EL). As propriedades destinadas à comparação de processos foram as tradicionalmente encontradas na literatura, as quais são elencadas no Quadro 2.

Quadro 2. Atributos de interesse dos processos de soldagem em aço inoxidável para o material do eixo naval

\begin{tabular}{|l|l|c|l|}
\hline \multicolumn{1}{|c|}{ Atributos } & Tipo & Objetivo & \multicolumn{1}{c|}{ Medida } \\
\hline Taxa de deposição (TD) & quantitativo & Max & $\mathrm{kg} / \mathrm{h}$ \\
\hline Energia de soldagem (E) & quantitativo & Min & $\mathrm{J} / \mathrm{mm}$ \\
\hline Rendimento econômico (R) & quantitativo & Max & proporção \\
\hline Diluição (D) & quantitativo & Min & Taxa \\
\hline Relação reforço-largura (EL) & quantitativo & Min & Taxa \\
\hline
\end{tabular}

A taxa de deposição, conforme definição de Modenesi [12], é a quantidade de material depositado por unidade de tempo e depende de vários fatores, incluindo o processo de soldagem, o tipo, o diâmetro e comprimento do eletrodo, a polaridade e o nível da corrente da soldagem, sendo calculada pela Equação 1:

$$
T D=\frac{P F-P I}{t}
$$

onde TD é taxa de deposição, PF é o peso final da chapa em $\mathrm{kg}$, PI é o peso inicial da chapa em kg, t é o tempo de arco aberto.

$A$ energia de soldagem (E) foi mensurada conforme a Equação 2

$$
E=\frac{(\eta * I * V)}{V_{S}}
$$

onde $\eta$ representa o rendimento térmico de cada processo; I representa a corrente; $V$ é a tensão; $V_{s}$ é a velocidade de soldagem.

O rendimento econômico (R) é definido por Modenesi [12] como a relação (em porcentagem) entre as taxas de deposição e de fusão conforme Equação 3. Se o 
eletrodo não possuir adições metálicas em seu revestimento, este parâmetro indica a proporção da alma que foi utilizada na formação da solda, conforme Equação 3:

$$
R=\frac{T D}{T f} 100
$$

onde TD é taxa de deposição e Tf, a taxa de fusão calculada pela Equação 4:

$$
T f=\frac{P f}{t}
$$

onde Pf é a massa de alma do eletrodo que foi fundida no tempo de arco aberto $(\mathrm{t})$ A penetração é a distância da superfície original do metal de base ao ponto em que termina a fusão, medida perpendicularmente a mesma.

A Relação reforço/largura é parte da análise da geometria da solda, sendo realizada pela avaliação dimensional da seção transversal da Figura 1 abaixo. As medidas de largura $(L)$, reforço $(R)$ e penetração $(P)$, definem tais características geométricas do cordão de solda depositado na superfície das peças metálicas [13].

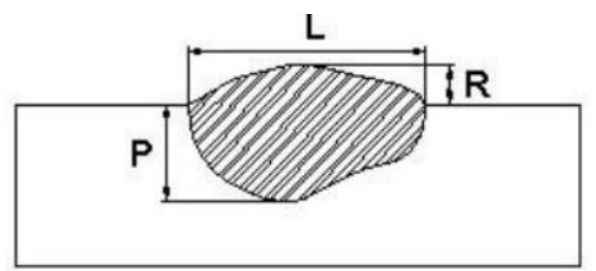

Figura 1. Representação das grandezas geométricas do cordão de solda

A diluição, ou "coeficiente de diluição, pode ser expressa pela Equação abaixo, onde "diluição" (ס), "A" e "B" são as áreas formadas na junta soldada [13].

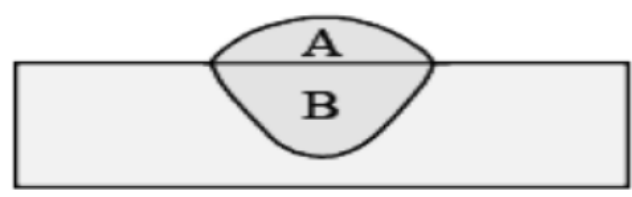

Figura 2. : Representação das áreas da junta soldada

As medidas diluição, penetração e relação reforço/largura definem as características geométricas do cordão de solda depositado na superfície de peças metálicas.

A Equação 5 define o percentual de diluição $(\delta)$ de cada processo.

onde $\mathrm{B}$ é a área da zona fundida e $\mathrm{A}$ representa a área do reforço.

$$
\delta=\frac{B}{B+A} 100
$$

A resistência mecânica é a deformação do material que gera a força resultante, na medida direta de seu módulo de elasticidade. Na engenharia, a resistência mecânica de uma estrutura é a sua capacidade de suportar as solicitações externas sem que estas venham a lhe causar deformações plásticas [11].

Neste estudo, foram aplicados seis métodos de atribuição de pesos que utilizam exclusivamente a ordem de prioridade informada pelo decisor, os quais são descritos a seguir.

O método que requer o menor conhecimento das preferências é de ponderação igual (equal weights - EW), segundo o qual os pesos são igualmente distribuídos conforme Equação 6 a seguir: 


$$
E W_{j}=\frac{1}{n}
$$

O método de ordenação recíproca (rank reciprocal weights - $R R$ ) utiliza as inversas das ordens dos atributos normalizados para cálculo dos respectivos pesos.

$$
R R_{j}=\frac{\frac{1}{r_{j}}}{\sum_{k=1}^{n} \frac{1}{r_{j}}}
$$

onde $R R_{j}$ e $r_{j}$ são respectivamente o peso e a posição do atributo $j, j=1, .2, . ., \mathrm{n}$.

O método da somatória (rank sum weights - RS) estima as ponderações por meio da seguinte equação:

onde $n$ é o total de atributos.

$$
R S_{j}=\frac{\left(n-r_{j}+1\right)}{\sum_{k=1}^{n}\left(n-r_{j}+1\right)}
$$

$O$ método de ordenação exponente (rank exponent weight - RE) é calculado conforme a seguinte fórmula:

$$
R E_{j}=\frac{\left(n-r_{j}+1\right)^{p}}{\sum_{k=1}^{n}\left(n-r_{j}+1\right)^{p}}
$$

na qual $p$ é um parâmetro que descreva a distribuição dos pesos entre os atributos e, conforme explica Roszkowska [9], quanto maior o valor de $p$, mais concentrado será o valor dos pesos em torno dos atributos tidos como mais importantes. O RE é uma generalização dos métodos $E W$ e $R S$, se $p=0$, RE equivale ao $E W$, e se $p=1$, equivale ao RS.

O método do centroide (rank order centroid - ROC), proposto por Barron e Barrett [14], estima os pesos que minimizam seus erros máximos a partir do centroide de todos os pesos possíveis, mantendo a ordem de importância dos atributos [8], conforme a equação a seguir.

$$
R O C=\frac{1}{n} \sum_{k=j}^{n} \frac{1}{r_{k}}
$$

A título de ilustração, tome-se um atributo na quarta posição de uma lista com o total de 5 , seu peso seria $R O C(4)=(0+0+0+1 / 4+1 / 5) / 5=0,09$, já o peso daquele que estivesse na quinta posição seria $\operatorname{ROC}(5)=(0+0+0+0+1 / 5) / 5=0,04$, como será exibido adiante.

Para simular o efeito da escolha dos pesos estimados, utilizou-se a Ponderação Aditiva Simples (Simple Additive Weighting - SAW) para avaliar os cinco processos de soldagem devido a sua simplicidade e por ser um dos métodos mais utilizados em problemas multicritérios [2].

O procedimento de cálculo do SAW inicia com a organização das medidas de desempenho das $m$ alternativas de processo numa matriz de decisão $A$.

$$
A=\left[x_{i j}\right]_{m \times n}
$$

onde $x_{i j}$ corresponde ao desempenho, ou utilidade, do processo $i$ associado ao atributo $j, i=1, \ldots, m$ e $j=1, \ldots, n$. 
Normalizam-se as medidas de desempenho conforme o atributo. Caso represente um objetivo ou propriedade que se deseja maximizar é denominado benefício, sendo normalizado por meio da Equação 11:

$$
a_{i j}=\frac{x_{i j}}{x_{j}^{\max }}
$$

Por ouro lado, se for um atributo cujo valor se deseja minimizar, denominado custo ou não-benefício, as medidas são normalizadas pela Equação 13:

$$
a_{i j}=\frac{x_{j}^{\min }}{x_{i j}}
$$

na qual $a_{i j}$ são as medidas de desempenho $x_{i j}$ normalizadas.

A função de utilidade de cada alternativa é calculada como a soma das medidas de desempenho normalizadas $\left(a_{i j}\right)$ ponderadas pelos pesos de seu respectivo atributo $\left(w_{j}\right)$, conforme Equação 14:

$$
S_{i}=\sum_{j=1}^{k} w_{j} a_{i j}
$$

Quanto maior a valor de $S_{i}$, maior a utilidade da alternativa, de modo que as alternativas podem ser ordenadas em ordem decrescente, sendo a preferível aquela com maior pontuação final.

\section{RESULTADOS E DISCUSSÃO}

Considerando-se que os processos de soldagem de revestimento em aço inoxidável AISI 316L se destinam a retificação em material do eixo propulsor naval, solicitou-se a um engenheiro mecânico experiente que organizasse as propriedades a serem avaliadas em ordem de importância. A partir dessa informação, foram estimados os pesos de cada atributo com base na ordenação por meio dos métodos atribuição de pesos, conforme as Equações de 6 a 10., sendo apresentados na Tabela 1.

Tabela 1. Estimativas de pesos dos atributos

\begin{tabular}{lcccccc}
\hline Atributos & TD & R & E & D & EL & \\
\hline Ordem & 1 & 2 & 3 & 4 & 5 & Amplitude \\
\hline EW & 0,2000 & 0,2000 & 0,2000 & 0,2000 & 0,2000 & 0,0000 \\
RR & 0,4380 & 0,2190 & 0,1460 & 0,1095 & 0,0876 & 0,3504 \\
RS & 0,3333 & 0,2667 & 0,2000 & 0,1333 & 0,0667 & 0,2666 \\
RE $(2)$ & 0,4545 & 0,2909 & 0,1636 & $0 /, 0727$ & 0,0182 & 0,4363 \\
RE $(3)$ & 0,5556 & 0,2844 & 0,1200 & 0,0356 & 0,0044 & 0,5512 \\
ROC & 0,4567 & 0,2567 & 0,1567 & 0,0900 & 0,0400 & 0,4167 \\
\hline
\end{tabular}

Nota: $E W=$ ponderação igual, $R R=$ ordenação recíproca, $R S=$ somatória; $R E(p)=$ exponente, sendo $p$ o valor do expoente, $R O C=$ centroide.

$\mathrm{O}$ método $\mathrm{RE}$ com parâmetro $\mathrm{p}=3$ foi que apresentou maior amplitude entre os atributos com maior e menor importância (amplitude $=0,55$ ). Os métodos com menores amplitudes foram o RS e o WE.

Numa situação em que o tomador de decisão somente é capaz de informar a ordem de prioridade dos atributos, a apresentação de uma tabela dessas estimativas seria útil uma vez que poderia tentar identificar entre as distribuições de pesos estimadas 
aquela que melhor se aproximasse de sua preferência, inserindo-as diretamente na análise, como será exemplificado mais adiante, ou adaptando-as para melhor ajuste.

Tabela 2. Matriz de decisão com as medidas de desempenho dos processos de soldagem

\begin{tabular}{cccccc}
\hline Processo & TD & R & E & D & EL \\
\hline Objetivo & Max & Max & Min & Min & Min \\
\hline SMAW & 0,4706 & 101,2658 & 167,3885 & 68,3930 & 0,1452 \\
GMAW 1 & 1,8444 & 127,2031 & 170,8100 & 64,8744 & 0,1901 \\
GMAW 2 & 0,8667 & 99,6169 & 227,8632 & 62,1556 & 0,2754 \\
GMAW 3 & 0,8667 & 99,6169 & 172,1281 & 64,5237 & 0,2377 \\
GMAW 4 & 1,6500 & 116,4706 & 167,2760 & 64,9214 & 0,2467 \\
GMAW 5 & 1,5800 & 111,5294 & 168,4540 & 66,1725 & 0,1827 \\
\hline Máximo & 1,8444 & 127,2031 & 227,8632 & 68,3930 & 0,2754 \\
Mínimo & 0,4706 & 99,6169 & 167,2760 & 62,1556 & 0,1452 \\
\hline
\end{tabular}

Tabela 3. Matriz normalizada

$\begin{array}{cccccc}\text { Processos } & \text { TD } & \text { R } & \text { E } & \text { D } & \text { EL } \\ \text { SMAW } & 0,2551 & 0,7961 & 0,9993 & 0,9088 & 1,0000 \\ \text { GMAW 1 } & 1,0000 & 1,0000 & 0,9793 & 0,1916 & 0,7637 \\ \text { GMAW 2 } & 0,4699 & 0,7831 & 0,7341 & 1,0000 & 0,5271 \\ \text { GMAW 3 } & 0,4699 & 0,7831 & 0,9718 & 0,9633 & 0,6107 \\ \text { GMAW 4 } & 0,8946 & 0,9156 & 1,0000 & 0,9574 & 0,5884 \\ \text { GMAW 5 } & 0,8566 & 0,8768 & 0,9930 & 0,9393 & 0,7946\end{array}$

Tabela 4. Pontuações finais de cada processo de soldagem obtidas pelo método SAW por método de ponderação.

\begin{tabular}{ccccccc}
\hline Processos & EW & RR & RS & RE(2) & RE(3) & ROC \\
\hline SMAW & 0,7919 & 0,6190 & 0,6850 & 0,5953 & 0,5248 & 0,5992 \\
GMAW 1 & 0,9402 & 0,9717 & 0,9745 & 0,9893 & 0,9950 & 0,9835 \\
GMAW 2 & 0,7028 & 0,6401 & 0,6808 & 0,6438 & 0,6098 & 0,6417 \\
GMAW 3 & 0,7598 & 0,6781 & 0,7290 & 0,6816 & 0,6374 & 0,6790 \\
GMAW 4 & 0,8712 & 0,8946 & 0,9092 & 0,9170 & 0,9141 & 0,9099 \\
GMAW 5 & 0,8921 & 0,8846 & 0,8962 & 0,8897 & 0,8814 & 0,8881 \\
\hline
\end{tabular}

Tabela 5. Posições de cada processo de soldagem obtidas pelo método SAW por método de ponderação.

\begin{tabular}{ccccccc}
\hline Processos & EW & RR & RS & RE(2) & RE(3) & ROC \\
\hline SMAW & 4 & 6 & 5 & 6 & 6 & 6 \\
GMAW 1 & 1 & 1 & 1 & 1 & 1 & 1 \\
GMAW 2 & 6 & 5 & 6 & 5 & 5 & 5 \\
GMAW 3 & 5 & 4 & 4 & 4 & 4 & 4 \\
GMAW 4 & 3 & 2 & 2 & 2 & 2 & 2 \\
GMAW 5 & 2 & 3 & 3 & 3 & 3 & 3 \\
\hline
\end{tabular}

As estimativas de pesos foram utilizadas para avaliação, pelo método SAW, dos cinco métodos de soldagem no procedimento de reparo do eixo propulsor naval em aço inoxidável AISI 316L, no intuído de ilustrar o efeito das estimativas de pesos sobre o resultado final de uma avaliação multicritério. 
As medidas de desempenho foram coletadas de um corpo de prova para cada método e são apresentadas na Tabela 2, juntamente com objetivo desejado para cada atributo.

A Tabela 3 exibe os valores dos desempenhos normalizados por meio das Equações 13 e 14, respectivamente, observados seus objetivos de maximização ou minimização, de forma a superar o obstáculo à comparação em decorrência de escalas diferentes.

Os resultados finais das avaliações são apresentados nas Tabelas 4 e 5, aquela com as pontuações finais $(S i)$ dos processos e esta com as posições alcançadas por cada processo considerando os pesos atribuídos às proriedades por cada método de estimação. Observa-se que o GMAW automatizado AWS AR 307 (GMAW 1), neste estudo, revelou-se a melhor alternativa para retificação de eixo naval em todas as distribuições de estimativas de pesos. De fato, o GMAW 1 obteve valores finais de utilidade bem acima dos demais, em média de 0,9757, enquanto que a segunda maior média foi 0,9027 (GMAW 4).

Todavia, é interessante notar que houve alterações de posições nos métodos EW, $\mathrm{RR}$ e RS, mais frequentemente nos dois primeiros, provavelmente em virtude de estimarem distribuições de pesos com menores amplitudes. Por exemplo, 0 processo SMAW, foi classificado em três posições diferentes e o processos GMAW 4 e GMAW 5 oscilaram entre a segunda e a terceira posição. Apesar de que tais alterações não se tenham verificado quando se aplicaram os métodos ER2, ER3 e ROC, não se pode afirmar que, em comparações em que as alternativas possuam desempenhos mais similares, tal estabilidade se mantenha.

\section{CONCLUSÃO}

Calcularam-se os pesos das propriedades destinadas à comparação de processos de soldagem utilizados no reparo de eixo propulsor naval de aço inoxidável AISI $316 \mathrm{~L}$ com base na ordenação fornecida pelo tomador de decisão, para tanto, foram realizadas seis estimativas de atribuição de pesos com base nas ordenações dos atributos a partir de cinco métodos.

Após a realização de mensuração dos desempenhos globais dos processos pelo método SAW utilizando tais estimativas, observou-se que a atribuição de pesos pode alterar a posição das alternativas o que pode determinar seleções diferentes, em especial, quando os métodos não geram estimativas muito dispersas, como o método de ponderação igual $(\mathrm{EW})$, em que não há dispersão, e método de ordenação recíproca (RR). Assim, a atribuição de pesos não é uma tarefa que possa ser considerada banal.

Sugere-se que, diante de uma situação em que o tomador de decisão é somente capaz de informar a ordem de prioridades dos atributos, elabore-se uma tabela ou gráficos com as distribuições de pesos dos métodos para que o mesmo possa tentar identificar aquela que se aproxime da melhor forma possível de suas preferências ou para que possa também ajustá-la antes de avançar para a fase de avaliação final das alternativas em análise.

\section{REFERÊNCIAS}

1 Statnikov R, Matusov J, Statnikov, A. Multicriteria Engineering Optimization Problems: statement, solution and applications. Journal of Optimization Theory and Applications. 2012, 155( 2):355-375. 
2 Hwang CL, Yoon K. Multiple Attribute Decision Making: methods and applications. New York: Springer-Verlag; 1981.

3 Jahan A, Mustapha F, Ismail MY, Sapuan, SM, Bahraminasab M. A comprehensive VIKOR method for material selection. Materials \& Design. 2011; 32(3): 1215-1221.

4 Biswas SA, Datta S, Bhaumik S, Majumdar G. Application of VIKOR Based Taguchi Method for Multi Response Optimization: a case study in submerged arc welding (SAW). In Proceedings of the International Conference on Mechanical Engineering; 2009 dez 26-28; Dhaka, Bangladesh, 2009;1-5.

5 Shyjith K, llangkumaran M, Kumanan S. Multi-criteria decision-making approach to evaluate optimum maintenance strategy in textile industry. Journal of Quality in Maintenance Engineering. 2008; 14(4): 375-386.

6 Vaidogas ER, Zavadskas EK, Turskis Z. Reliability measures in multicriteria decision making as applied to engineering projects. International Journal of Management and Decision Making. 2007; 8(5-6): 497-518.

7 Capraz O, Meran C, Wörner W, Gungor A. Using AHP and TOPSIS to evaluate welding processes for manufacturing plain carbon stainless steel storage tank. Archives of Materials Science. 2015; 76(2): 156-162.

8 Caprace, JD, Rigo, P (2009, May). Multi-criteria decision support for cost assessment techniques in shipbuilding industry. In Proc. of Conference COMPIT'09, 10-12 Mai 2009 (pp. 6-21).

9 Roszkowska, E (2013). Rank Ordering Criteria Weighting Methods - a comparative overview Optimum. Studia Ekonomiczne; 5(65): 14-33.

10 Buede DM, Miller, WD. The engineering design of systems: models and methods. New York: John Wiley \& Sons; 2016.

11 Jahan A, Edwards KL, Bahraminasab M. (2016). Multi-criteria decision analysis for supporting the selection of engineering materials in product design. ButterworthHeinemann; 2016.

12 Modenesi PJ. Técnica operatória da soldagem SAW, UFMG/DEMM. [acesso em 30 mai. 2017]. Disponível em: http://www.infosolda.com.br/artigos/prosol04.pdf.

13 Assunção PDAC. Estudo da viabilidade do processo de soldagem GMAW-DCW (DOUBLE COLD WIRE) [dissertação de mestrado]. Pará: Universidade Federal do Para; 2013.

14 Barron FH, Barrett BE. Decision quality using ranked attribute weights. Management Science. 1996; 42(11): 1515-1523. 